DOI: $10.2478 /$ awutm-2014-0003

DE GRUYTER

OPEN
Analele Universităţii de Vest,

Timişoara

Seria Matematică - Informatică

LII, 1, (2014), 29- 39

\title{
Generalized Jensen functional equation on restricted domain
}

Abdellatif Chahbi*, Muaadh Almahalebi, Ahmed Charifi and Samir Kabbaj

Abstract. We prove the Hyers-Ulam stability on restricted domains of generalized Jensen functional equation

$$
\sum_{k=0}^{m-1} f\left(x+b_{k} y\right)=m f(x), x, y \in E,
$$

where $b_{k}=\exp \left(\frac{2 i \pi k}{m}\right)$ for $0 \leq k \leq m-1$. These results are applied to study of an asymptotic behavior of these functional equation.

AMS Subject Classification (2000). Primary 39B82; Secondary 39B52.

Keywords. Jensen functional equation, K-quadratic functional equation, Pexider functional equation, Hyers-Ulam stability.

\section{Introduction}

The question concerning the stability of functional equations has been first raised by S. M. Ulam in 1940 [38]. S. M. Ulam started the stability by the following question

Given a group $G$, a metric group $\left(G^{\prime}, d\right)$, a number $\delta>0$ and a mapping $f: G \longrightarrow G^{\prime}$ which satisfies the inequality $d(f(x y), f(x) f(y))<\delta$ for all 
$x, y \in G$, does there exist an homomorphism $h: G \longrightarrow G^{\prime}$ and a constant $\gamma>0$, depending only on $G$ and $G^{\prime}$ such that $d(f(x), h(x)) \leq \gamma$ for all $x$ in $G$ ?

In 1941, Ulam's problem for the case of approximately additive mappings was solved by D. H. Hyers [17] on Banach spaces. In 1950 T. Aoki [2] provided a generalization of the Hyers theorem for additive mappings and in 1978 Th. M. Rassias [32] generalized the Hyers theorem for linear mappings by considering an unbounded Cauchy difference. For more information on the concept of the stability of functional equations see for example [7],[12],[16],[18],[19],[20],[23],[26].

The first stability theorem for the K-quadratic functional equation was proved in 1941 by Hyers-Ulam [17] and in 1978 by Th.M. Rassias [32] for $K=\{i d\}$ then by F. Skof in 1983 [37] for $K=\{-i d, i d\}$ in Banach spaces. In 1984, P.W. Cholewa [11] extended Skof's result to an abelian group. In 1992, S. Czerwik [13], in the spirit of Hyers-Ulam- Rassias generalized the Skof's theorem.

Recently, the stability problem of the K-quadratic functional equation has been investigated by a number of mathematicians, the interested reader should refer to Ait Sibaha, Bouikhalene and Elqorachi [1], B.Bouikhalene, et al. [5], A.Charifi, et al. [10], Ab.Chahbi, et al. [9] and R. Łukasik [27], see also [6],[20],[22]-[25] and [32].

In 1998 Jung [22] investigated the Hyers-Ulam stability for additive and quadratic mappings on restricted domains. In 2002, J. M. Rassias [36] improved the bounds and thus the stability results obtained by Jung. Besides, he establish the Ulam stability for more general equations of two types on a restricted domain. Finally, he apply our recent results to the asymptotic behavior of functional equations of different types.

The stability problems of several functional equations on a restricted domain have been extensively investigated by a number of authors, for example ([8], [15], [30] and [31]).

Throughout this paper, let $E, F$ be vector spaces over $K=\mathbb{Q}$ or $\mathbb{R}$ and $2 \leq m \in \mathbb{N}$. Our aim is to prove the Hyers-Ulam stability on restricted domains of generalized Jensen functional equation

$$
\sum_{k=0}^{m-1} f\left(x+b_{k} y\right)=m f(x), x, y \in E
$$

where $b_{k}=\exp \left(\frac{2 i \pi k}{m}\right)$ for $0 \leq k \leq m-1$. These results are applied to study of an asymptotic behavior of these functional equation. 


\section{$2 \quad$ Notations and Preliminary results}

In this section, we need to introduce some notions and notations.

A function $A: E \rightarrow F$ between two vector spaces $E$ and $F$ is said to be additive provided if $A(x+y)=A(x)+A(y)$ for all $x, y \in E$. In this case, it is easily seen that $A(r x)=r A(x)$ for all $x \in E$ and all $r \in \mathbb{Q}$.

Let $k \in \mathbb{N}$ and $A: E^{k} \rightarrow F$ be a function, then we say that $A$ is $k$-additive provided if it is additive in each variable. In addition, we say that $A$ is symmetric provided if

$$
A\left(x_{\sigma(1)}, x_{\sigma(2)}, \ldots, x_{\sigma(k)}\right)=A\left(x_{1}, x_{2}, \ldots, x_{k}\right)
$$

whenever $x_{1}, x_{2}, \ldots, x_{k} \in E$ and $\sigma$ is a permutation of $(1,2, \ldots, k)$.

Let $k \in \mathbb{N}$ and $A: E^{k} \rightarrow F$ be symmetric and $k$-additive and let $A_{k}(x)=$ $A(x, x, \ldots, x)$ for $x \in E$ and note that $A_{k}(r x)=r^{k} A_{k}(x)$ whenever $x \in E$ and $r \in \mathbb{Q}$.

In this way a function $A_{k}: E \longrightarrow F$ which satisfies for all $\lambda \in \mathbb{Q}$ and $x \in E$, $A_{k}(\lambda x)=\lambda^{k} A_{k}$ will be called a rational-homogeneous form of degree $k$, (assuming $A_{k} \neq 0$ ).

A function $p: E \rightarrow F$ is called a generalized polynomial (GP) function of degree $m \in \mathbb{N}$ if there exist $a_{0} \in E$ and a rational-homogeneous form $A_{k}: E \rightarrow F$ (for $1 \leq k \leq m$ ) of degree $k$, such that

$$
p(x)=a_{0}+\sum_{k=1}^{m} A_{k}(x)
$$

for all $x \in E$.

Let $F^{E}$ denote the vector space, over a field $K$, consisting of all maps from $E$ into $F$. For each $h \in E$, define the linear difference operator $\Delta_{h}$ on $F^{E}$ by

$$
\Delta_{h} f(x)=f(x+h)-f(x)
$$

for all $f \in F^{E}$ and all $x \in E$. Notice that these difference operators commute $\left(\Delta_{h_{1}} \Delta_{h_{2}}=\Delta_{h_{2}} \Delta_{h_{1}}\right.$ for all $\left.h_{1}, h_{2} \in E\right)$ and if $h \in E$ and $n \in \mathbb{N}$, then $\Delta_{h}^{n}$ the $n$-th iterate of $\Delta_{h}$ satisfies

$$
\Delta_{h}^{n} f(x)=\sum_{k=0}^{n}(-1)^{n-k}\left(\begin{array}{l}
n \\
k
\end{array}\right) f(x+k h)
$$

for all $f \in F^{E}$ and all $x, h \in E$.

The following theorem is proved by Mazur and Orlicz [28],[29] then in greater generality by Djokovic [14]. 
Theorem 2.1. Let $n \in \mathbb{N}$ and $f: E \rightarrow F$ be a function between two vector spaces $E$ and $F$, then the following assertions are equivalent.

1. $\Delta_{h}^{n} f(x)=0$ for all $x, h \in E$.

2. $\Delta_{h_{n}} \ldots \Delta_{h_{1}} f(x)=0$ for all $x, h_{1}, \ldots, h_{n} \in E$.

3. $f$ is a generalized polynomial (GP) of degree at most $n-1$.

\section{Main results}

Lemma 3.1. Suppose that $2 \leq m \in \mathbb{N}, b_{k} \in K$ for $0 \leq k \leq m, F$ is a real (or complex) Banach space, $\delta \geq 0$ and $f_{k}: E \rightarrow F$ for $0 \leq k \leq m$, be mappings fulfilling

$$
\left\|\sum_{k=0}^{m} f_{k}\left(x+b_{k} y\right)-f_{k}(x)\right\| \leq \delta
$$

for all $x, y \in E$ and assume that $b_{k}-b j \neq 0$ and $b_{0} \neq 0$ whenever $0 \leq j<$ $k \leq m$. Then,

$$
\left\|\Delta_{h_{m+1}} \ldots \Delta_{h_{1}} f_{0}(x)\right\| \leq 3^{m} \delta
$$

for all $x, h_{1}, \ldots, h_{m+1} \in E$.

Proof. For $0 \leq k \leq m$ let $d_{j k}=b_{k}-b_{j}$ so that $d_{j k}=0$ if $j<k$ and $d_{k k}=0$. For $0 \leq k \leq m$ and $x, y, h_{1} \in E$,

$$
\left(x-b_{m} h_{1}\right)+b_{k}\left(y+h_{1}\right)=x+b_{k} y+d_{k m} h_{1} .
$$

By using these quality and (3.1), we find that

$$
\begin{gathered}
\left\|\sum_{k=0}^{m}\left(f_{k}\left(x+b_{k} y+d_{k m} h_{1}\right)-f\left(x+b_{k} y\right)\right)-\sum_{k=0}^{m}\left(f_{k}\left(x+d_{k m} h_{1}\right)-f_{k}(x)\right)\right\| \\
\leq\left\|\sum_{k=0}^{m} f_{k}\left(x+b_{k} y+d_{k m} h_{1}\right)-f_{k}\left(x-b_{m} h_{1}\right)\right\|+\left\|\sum_{k=0}^{m} f_{k}\left(x+d_{k m} h_{1}\right)-f_{k}\left(x-b_{m} h_{1}\right)\right\| \\
+\left\|\sum_{k=0}^{m} f_{k}\left(x+b_{k} y\right)-f_{k}(x)\right\| \leq 3 \delta
\end{gathered}
$$


and thus, since $d_{m m}=0$,

$$
\left\|\sum_{k=0}^{m-1} \Delta_{d_{k m} h_{1}} f_{k}\left(x+b_{k} y\right)-f_{k}(x)\right\| \leq 3 \delta
$$

for all $x, y, h_{1} \in E$. Repeating the argument that led from (3.1) to (3.3), we find that

$$
\left\|\sum_{k=0}^{m-2} \Delta_{d_{k, m-1} h_{2}} \Delta_{d_{k m} h_{1}} f_{k}\left(x+b_{k} y\right)-f_{k}(x)\right\| \leq 3 \delta
$$

for all $x, y, h_{1}, h_{2} \in E$. Applying this reasoning $m-2$ more times we are inclined to admit that

$$
\left\|\Delta_{d_{01} h_{m}} \ldots \Delta_{d_{0 m} h_{1}} f_{0}\left(x+b_{0} y\right)-f_{k}(x)\right\| \leq 3^{m} \delta
$$

for all $x, y, h_{1}, \ldots h_{m} \in E$. Consequently,

$$
\left\|\Delta_{x} \Delta_{d_{01} h_{m}} \ldots \Delta_{d_{0 m} h_{1}} f_{0}\left(b_{0} y\right)\right\| \leq 3^{m} \delta .
$$

Since $d_{0 k} \neq 0$ for $1 \leq k \leq m$, and $b_{0} \neq 0$ the last inequality simplify asserts that

$$
\left.\| \Delta_{h_{m+1}} \ldots \Delta_{h_{1}} f_{0}(z)\right) \| \leq 3^{m} \delta
$$

for all $z, h_{1}, \ldots, h_{m+1} \in E$.

Theorem 3.2. Suppose that $E$ is a vector space and $F$ is real (or complex) Banach space, $2 \leq m \in \mathbb{N}, b_{k}=\exp \left(\frac{2 i k \pi}{m}\right)$ for $0 \leq k \leq m-1$ and $\delta \geq 0$. If $f: E \rightarrow F$ satisfies

$$
\left\|\sum_{k=0}^{m-1} f\left(x+b_{k} y\right)-m f(x)\right\| \leq \delta
$$

for all $x, y \in E$. Then there exists a unique generalized polynomial (GP) $q: E \rightarrow F$ at most $m-1$ such that $q(0)=0$ and

$$
\|f(x)-f(0)-q(x)\| \leq 3^{m-1} \delta \text { for all } x \in E .
$$

Moreover

$$
\sum_{k=1}^{m} q\left(x+b_{k} y\right)-m q(x)=0 \text { for all } x, y \in E
$$


Proof. If we put $f_{k}=f$ for each $k$, in (3.8), we get

$$
\left\|\sum_{k=0}^{m-1} f_{k}\left(x+b_{k} y\right)-f_{k}(x)\right\| \leq \delta
$$

Then, by Lemma 3.1

$$
\left\|\Delta_{h_{m}} \ldots \Delta_{h_{1}} f(z)\right\| \leq 3^{m-1} \delta
$$

for all $z, h_{1}, \ldots, h_{m} \in E$.

Hence, according to (Theorem II [20]), there exists a generalized polynomial (GP) $q: E \rightarrow F$, of degree at most $m-1$ such that

$$
\|f(x)-q(x)\| \leq 3^{m-1} \delta
$$

and

$$
q(x)=f(0)+\sum_{j=1}^{m-1} A_{j}(x)
$$

where $A_{j}: E^{j} \rightarrow F$ are symmetric, $j$-additive mappings.

By (3.10) and (3.11), we obtain

$$
\left\|\sum_{k=0}^{m-1}\left(q\left(x+b_{k} y\right)\right)-m q(x)\right\| \leq\left\|\sum_{k=0}^{m-1}\left(q\left(x+b_{k} y\right)-f\left(x+b_{k} y\right)\right)\right\|+\|m f(x)-m q(x)\|
$$

so,

$$
\left\|\sum_{k=0}^{m-1}\left(q\left(x+b_{k} y\right)\right)-m q(x)\right\| \leq 2 m \cdot 3^{m-1} \delta
$$

for all $x, y \in E$.

Now (3.12) says, in light of (3.11), that

$$
\left\|\sum_{j=1}^{m-1}\left(\sum_{k=0}^{m-1} A_{j}\left(x+b_{k} y\right)-m A_{j}(x)\right)\right\| \leq 2 m \cdot 3^{m-1} \delta
$$

for all $x, y \in E$. In (3.13), replace $x$ by $r x$ and $y$ by $r y(r \in \mathbb{Q})$ to conclude that, for all $x, y \in E$ and all $r \in \mathbb{Q}$,

$$
\left\|\sum_{j=1}^{m-1} r^{j}\left(\sum_{k=0}^{m-1} A_{j}\left(x+b_{k} y\right)-m A_{j}(x)\right)\right\| \leq 2 m .3^{m-1} \delta
$$


By continuity, (3.14) holds for all real $r$, and all $x, y \in E$. Now suppose that $\phi: F \rightarrow \mathbb{R}$ is a continuous linear functional. Then

$$
\left\|\sum_{j=1}^{m-1} r^{j} \phi\left(\sum_{k=0}^{m-1}\left(A_{j}\left(x+b_{k} y\right)\right)-m A_{j}(x)\right)\right\| \leq\|\phi\| 2 m .3^{m-1} \delta
$$

for all $x, y \in E$ and all $r \in \mathbb{R}$. Since a real polynomial function is bounded if and only if it is constant, from the last inequality we surmise that, for $1 \leq j \leq m-1$,

$$
\phi\left(\sum_{k=0}^{m-1} A_{j}\left(x+b_{k} y\right)-m A_{j}(x)\right)=0
$$

for all $x, y \in E$. Since this is so for every continuous linear functional $\phi$ : $F \rightarrow \mathbb{R}$, by the Hahn- Banach theorem,

$$
\sum_{k=0}^{m-1} A_{j}\left(x+b_{k} y\right)-m A_{j}(x)=0 \text { for } x, y \in E \text { and } 1 \leq j \leq m-1 .
$$

Then, each $q$ is a generalized polynomial (GP) of degree at most $m-1$ and from (3.17) we find that

$$
\sum_{k=0}^{m-1} q\left(x+b_{k} y\right)-m q(x)=0 \text { for } x, y \in E \text { and } 1 \leq j \leq m-1 .
$$

Finally, let $p$ be another generalized polynomial (GP) solution of (1.1) of degree at most $m-1$ such that

$$
\|f(x)-f(0)-p(x)\| \leq 3^{m-1} \delta, x \in E,
$$

and $p(0)=0$. Then, from (3.18) and (3.10) we get that $q-p$ is generalized polynomial (GP) of degree at most $m-1$ such that $\|q(x)-p(x)\| \leq$ $2.3^{m-1}, x \in E$. Thus, necessarily $q=p+q(0)-p(0)$. Since $q(0)=p(0)=0$ we get that $q=p$ witch ends the proof.

Theorem 3.3. Let $d>0$ and $\delta \geq 0$ be fixed. If a mapping $f: E \rightarrow F$ satisfies the generalized Jensen inequality (3.8) for all $x, y \in E$, with $\|x\|+$ $\|y\| \geq d$, then there exists a unique generalized polynomial (GP) $q: E \rightarrow F$ such that $q(0)=0$ and

$$
\|f(x)-f(0)-q(x)\| \leq 3^{m} \delta \text { for all } x \in E .
$$

Moreover

$$
\sum_{k=0}^{m-1} q\left(x+b_{k} y\right)-m q(x)=0 \text { for all } x, y \in E
$$


Proof. Assume that $\|x\|+\|y\|<d$ and let $t \in E$ such that $\|t\|=d$ where $x=0$ and

$$
t=\left(1+\frac{d}{\|x\|}\right) x
$$

where $x \neq 0$. We note that $\|t\|=\|x\|+d \geq d,\|x\|+\|t\| \geq d$ and

$$
\|t\|+\left\|x+b_{k} y\right\| \geq d
$$

We have for all $b_{j}, 0 \leq j \leq m-1$ is a root of unity, if we put $b_{j}=e^{i \theta}$, we obtain

$$
\left\|x+b_{j} t\right\|^{2}=\left(2\left(1+\frac{d}{\|x\|}\right)(1+\cos \theta)+\frac{d^{2}}{\|x\|^{2}}\right)\|x\|^{2} \geq d^{2}
$$

Thus, from (3.8) and the new functional identity we get

$$
\begin{aligned}
m\left[\sum_{k=0}^{m-1} f\left(x+b_{k} y\right)-m f(x)\right] & =\left[\sum_{k=0}^{m-1}\left(m f\left(x+b_{k} y\right)-\sum_{n=0}^{m-1} f\left(x+b_{k} y+b_{n} t\right)\right)\right] \\
& +\left[\sum_{n=0}^{m-1}\left(-m f\left(x+b_{n} t\right)+\sum_{k=0}^{m-1} f\left(x+b_{n} t+b_{k} y\right)\right)\right] \\
& +m\left[\sum_{n=0}^{m-1} f\left(x+b_{n} t\right)-m f(x)\right],
\end{aligned}
$$

then

$$
m\left\|\sum_{k=0}^{m-1} f\left(x+b_{k} y\right)-m f(x)\right\| \leq 3 m \delta
$$

Therefore,

$$
\left\|\sum_{k=0}^{m-1} f\left(x+b_{k} y\right)-m f(x)\right\| \leq 3 \delta .
$$

for all $x, y \in E$. Now, Applying Theorem 3.2 we get the result.

Corollary 3.4. A mapping $f: E \rightarrow F$ satisfies the functional equation (1.1) if and only if the asymptotic condition

$$
\left\|\sum_{k=0}^{m-1} f\left(x+b_{k} y\right)-m f(x)\right\| \rightarrow 0, \text { as }\|x\|+\|y\| \rightarrow \infty .
$$

holds true. 
By the asymptotic condition (3.19), there exists a sequence $\delta_{n}$ monotonically decreasing to 0 such that

$$
\left\|\sum_{k=0}^{m-1} f\left(x+b_{k} y\right)-m f(x)\right\| \leq \delta_{n}
$$

for all $x, y \in E$ with $\|x\|+\|y\| \geq n$. Hence, it follows from Theorem 3.3 that there exists a unique generalized polynomial $q_{n}: E \rightarrow F$ such that $q_{n}(0)=0$ and

$$
\left\|f(x)-f(0)-q_{n}(x)\right\| \leq 3^{m} \delta_{n} \text { for all } x \in E .
$$

for all $x \in E$. Since $\delta_{n}$ is a monotonically decreasing sequence, the generalized polynomial $q_{n}$ satisfies (3.21) for all $N \geq n$. The uniqueness of $q_{n}$ implies for all $N \geq n, q_{N}=q_{n}$. Hence, by letting $n \rightarrow \infty$, in (3.21) we conclude that $f=f(0)+q_{N}$ is generalized polynomial of degree at most $m-1$, and it satisfied the functional equation (1.1).

\section{Acknowledgement}

We are greatly indebted to the referee for valuable suggestions that led to an overall improvement of the paper.

\section{References}

[1] M. Ait Sibaha; B. Bouikhalene and E. Elqorachi, Hyers-Ulam-Rassias stability of the $K$-quadratic functional equation, . Ineq. Pure and appl. Math., 8, (2007), article 89

[2] T. Aoki, On the stability of the linear transformation in Banach spaces, J. Math. Soc. Japan, 2, (1950), 64-66

[3] L.M. Arriola and W.A. Beyer, Stability of the Cauchy functional equation over p-adic fields, Real Analysis Exchange, 31, (2005/2006), 125-132

[4] J. A. Baker, A general functional equation and its stability, Proceeding of the American Mathematical Society, 133, Number 6, (2005), 1657-1664

[5] B. Bouikhalene; E. Elqorachi and Th.M. Rassias, On the generalized HyersUlam stability of the quadratic functional equation with a general involution, Anal. Appl. Appl., 11, (2008), 805-818

[6] N. Brillouet-Belluot; J. Brzdȩk and K. Cieplinski, On some recent developments in Ulam's type stability, Abstr. Appl. Anal., (2012), Art. ID 716936, 41 pp. 
[7] J. Brzdęk, Hyperstability of the Cauchy equation on restricted domains, Acta Math. Hungar., 141, (2013), 58-67

[8] J. Brzdȩk, On a method of proving the Hyers-Ulam stability of functional equations on restricted domains, Austr. J. Math. Anal. Appl., 6, (2009), 1-10

[9] AB.Chahbi; A.Charifi; B. Bouikhalene and S. Kabbaj, Operatorial approach to the non-Archimedean stability of a Pexider K-quadratic functional equation, Arab Journal of Mathematical Sciences, (2014)

[10] A. Charifi; B. Bouikhalene; E. Elqorachi and A. Redouani, Hyers-UlamRassias stability of a generalized Jensen functional equation, The Australian Journal of Mathematical Analysis and Applications, 6, (2009), Issue 1, Article 19, pp. 1-16

[11] P.W. Cholewa, Remarks on the stability of functional equtaions, Aequationes Math., 27, (1984), 76-86

[12] K. Cieplinski, Applications of fixed point theorems to the Hyers-Ulam stability of functional equations-a survey, Ann. Funct. Anal., 3 no. 1, (2012), 151-164

[13] S. Czerwik, On the stability of the quadratic mapping in normed spaces, Abh. Math. Sem. Univ. Hamburg, 62, (1992), 59-64

[14] D.Z. Djokovic, A representation theorem for $(X 1-1)(X 2-1)(X n-1)$ and its applications, Ann. Polon. Math., 22, (1969), 189-198. MR0265798 (42:707)

[15] E. Elqorachi and Y. Manar, On the paper by A. Najati and S.-M. Jung: The HyersUlam stability of approximately quadratic mapping on restricted domains, Journal of Nonlinear Analysis and Application, 2012, (2012)

[16] P. Găvrută, A generalization of the Hyers-Ulam-Rassias stability of approximately additive mappings, J. Math. Anal. Appl., 184, (1994), 431-436

[17] D.H. Hyers, On the stability of the linear functional equation, Proc. Nat. Acad. Sci. U.S.A., 27, (1941), 222-224

[18] D.H. Hyers and Th.M. Rassias, Approximate homomorphisms, Aequationes Math., 44, (1992), 125-153

[19] D.H. Hyers; G.I. Isac and Th.M. Rassias, Stability of Functional Equations in Several Variables, Birkhäuser, Basel, (1998)

[20] D.H. Hyers, Transformations with bounded n-th differences, Pacific J. Math., 11, (1961), 591-602. MR0132401 (24:A2246)

[21] K.-W. Jun and Y.-H. Lee, A generalization of the Hyers-Ulam-Rassias stability of Jensen's equation, J. Math. Anal. Appl., 238, (1999), 305-315

[22] S.M. Jung, On the Hyers-Ulam stability of the functional equations that have the quadratic property, J. Math. Anal. Appl., 222, (1998), 126-137

[23] S.M. Jung, Stability of the quadratic equation of Pexider type, Abh. Math. Sem. Univ. Hamburg, 70, (2000), 175-190

[24] S.M. Jung and P. K. Sahoo, Hyers-Ulam stability of the quadratic equation of Pexider type, J. Korean Math. Soc., 38 No. 3, (2001), 645-656

[25] C.F.K. Jung, On generalized complete metric spaces, Bull. A.M.S., 75, (1969), $113-116$ 
[26] Y. Li and L. Hua, Hyers-Ulam stability of polynomial equation, Banach J. Math. Anal., 3 no. 2, (2009), 86-90

[27] R. Lukasik, Some generalization of Cauchy's and the quadratic functional equations, Aequat. Math., 83, (2012), 75-86

[28] S. Mazur and W. Orlicz, Grundlegende Eigenschaften der Polynomischen Operationen, Erst Mitteilung, Studia Math., 5, (1934), 50-68

[29] S. Mazur and W. Orlicz, Grundlegende Eigenschaften der Polynomischen Operationen, Zweite Mitteilung, ibidem, 5, (1934), 179-189

[30] A.Najati and S. M.Jung, Approximately quadratic mappings on restricted domains, J. Ineq. Appl, (2010)

[31] A. Rahimi and A. Najati, On the asymptoticity aspect of Hyers-Ulam stability of quadratic mappings, Journal of Inequalities and Applications, vol. 2010, (2011)

[32] Th.M. Rassias, On the stability of linear mapping in Banach spaces, Proc. Amer. Math. Soc., 72, (1978), 297-300

[33] Th.M. Rassias, On the stability of the functional equations and a problem of Ulam, Acta Applicandae Mathematicae, 62, (2000), 23-130

[34] and P. Šemrl Th.M. Rassias, On the behavior of mappings which do not satisfy Hyers-Ulam stability, Proc. Amer. Math. Soc., 114, (1992), 989-993

[35] Th.M. Rassias and J. Tabor, Stability of Mappings of Hyers-Ulam Type, Hardronic Press, Inc., Palm Harbor, Florida, (1994)

[36] J.M. Rassias, On the Ulam stability of mixed type mappings on restricted domains, J. Math. Anal. Appl., 276, (2002), 747-762

[37] F. Skof, Local properties and approximations of operators, Rend. Sem. Mat. Fis. Milano, 53, (1983), 113-129

[38] S.M. Ulam, A Collection of Mathematical Problems, Interscience Publ. New York, 1961. Problems in Modern Mathematics, Wiley, New York, (1964)

Abdellatif Chahbi*, Muaadh Almahalebi, Ahmed Charifi and Samir Kabbaj

Department of Mathematics

Faculty of Sciences

University of Ibn Tofail

Kenitra, Morocco

E-mail: ab_1980@live.fr, muaadh1979@hotmail.fr, charifi2000@yahoo.fr and samkabbaj@yahoo.fr

Received: 06.02.2014

Accepted: 10.06.2014 